\title{
SHARP NORM INEQUALITIES FOR THE TRUNCATED HILBERT TRANSFORM
}

\author{
ENRICO LAENG
}

Abstract. We split the classical Hilbert Transform $H$ into the sum of two convolution integrals $H^{(\delta)}+R^{(\delta)}$, where the kernel of $H^{(\delta)}$ is supported away from the origin in $\{|t| \geqslant \delta\}$, while the kernel of $R^{(\delta)}$ is supported near the origin in $\{|t| \leqslant \delta\}$. We prove that the $L^{p}$-norm of $H^{(\delta)}$, known in the literature as the Truncated Hilbert Transform, is equal to the norm of $H$. Namely $\left\|H^{(\delta)}\right\|_{p, p}=\cot (\pi / 2 p)$ for $2 \leqslant p<+\infty$, and $\left\|H^{(\delta)}\right\|_{p, p}=\tan (\pi / 2 p)$ for $1<p \leqslant 2$. We then prove that the $L^{p}$-norm of $R^{(\delta)}$ is stricly larger. In particular $\left\|R^{(\delta)}\right\|_{2,2}=1.17897 \ldots$, a constant related to the Gibbs phenomenon.

Mathematics subject classification (2000): 42A45, 42A50, 41A44.

Keywords and phrases: Truncated Hilbert transform, $L^{p}$ norm, best constants.

\section{REFERENCES}

[1] L. De Carli and E. Laeng, Sharp $L^{p}$ estimates for the segment multiplier, Collect. Math., 51, 3 (2000), 309-326.

[2] L. De Carli and E. Laeng, On the $(p, p)$ norm of monotonic Fourier multipliers, C. R. Acad. Sci. Paris, 330, Série I (2000), 657-662.

[3] L. GrafaKos, Best bounds for the Hilbert transform on $L^{p}\left(\mathbb{R}^{1}\right)$, Math. Res. Lett., 4 (1997), 469-471.

[4] E. LAENG, Remarks on the Hilbert Transform and on some families of multiplier operators related to it, Collect. Math., 58, 1 (2007), 25-44.

[5] W. McLean And D. Elliott, On the p-norm of the truncated Hilbert Transform, Bull. of the Australian Math. Society, 38 (1988), 413-420.

[6] S. K. Pichorides, On the best values of the constants in the theorems of M. Riesz, Zygmund and Kolmogorov, Studia Math., 44 (1972), 165-179.

[7] A. Zygmund, Trigonometric series. Vol. I, II., Reprint of the 1979 edition, Cambridge Mathematical Library, Cambridge University Press. 